\title{
Experimental and Numerical Study on the Flow Characteristics of Synthetic Jets*
}

\author{
Koichi NISHIBE**, Yuki FUJITA**, Kotaro SATO***, Kazuhiko YOKOTA**** \\ and Toru $\mathrm{KOSO}^{* * * * *}$ \\ **Graduate School of Mechanical Engineering, Kogakuin University, \\ Nishi-Shinjuku 1-24-2, Shinjuku-ku, Tokyo 163-8677, Japan \\ E-mail: knishibe@ykh.chiyoda.co.jp

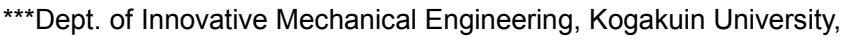 \\ Nishi-Shinjuku 1-24-2, Shinjuku-ku, Tokyo 163-8677, Japan \\ ****Dept. of Mechanical Engineering, Aoyamagakuin University, \\ Fuchinobe 5-10-1, Chuo-ku, Sagamihara-shi, Kanagawa 252-5258, Japan \\ *****Interdisciplinary Graduate School of Engineering Sciences, Kyushu University, \\ Kasuga-koen 6-1, Kasuga-shi, Fukuoka 816-8580, Japan
}

\begin{abstract}
Over the last decade, several studies have investigated synthetic jets. However, there are still many clarifications needed, including details of the structure and Coanda effect of synthetic jets. The present study clarifies some fundamental flow characteristics of free synthetic jets and synthetic jets near a rigid boundary by conducting an experiment and numerical simulations. As the main results, it is found that the velocity distribution of free synthetic jets depends on $K=\operatorname{Re} / S^{2}$ (the ratio of the Reynolds number to the square of the Stokes number) and can be identified by the maximum velocity at the centerline and the jet half-width. In addition, it is confirmed that the flow characteristics of the synthetic jet near a rigid boundary and the re-attachment length of the synthetic jet are determined not only by $H_{I} / b_{0}$ (normalized step height) but also $K$.
\end{abstract}

Key words: Synthetic Jet, Continuous Jet, Reynolds Number, Stokes Number, Velocity Distribution, Turbulence, Numerical Simulation

\section{Introduction}

Jet flows have been applied in various fields to control flow separation. In many cases, a continuous jet is generated by heavy turbo machines, such as a centrifugal compressor that consists of many parts. Therefore, it is difficult to design a downsized flow control system. As an alternative to continuous jets, synthetic jets can be generated by a compact actuator (e.g., piezoelectric actuator, speaker-driven actuator) [1] - [7].

Over the last decade, several experimental and numerical studies have focused on synthetic jets. The onset conditions and the structure of the synthetic jet have been discussed. For example, Holman et al. [2] indicated that synthetic jet formation is governed by $K=R e / S^{2}$ (the ratio of the Reynolds number to the square of the Stokes number). Koso and Kinoshita [7] investigated the difference of the flow characteristics (e.g., velocity profile, entrainment, momentum) between continuous jets and synthetic jets. However, there are still many details to be explained, including the jet structure and the unsteady flow characteristics. Especially, the Coanda effect of a synthetic jet near a rigid plane boundary has not been thoroughly investigated.

${ }^{*}$ Received 13 Jan., 2011 (No. 11-0028) [DOI: 10.1299/jfst.6.425]

Copyright $\odot 2011$ by JSME 
The present study uses an experiment and numerical simulations to clarify some of the fundamental flow characteristics of free synthetic jets and synthetic jets near a rigid boundary. To clarify the Coanda effect of synthetic jets, the influence of a rigid plane boundary on the behavior of synthetic jets with various $K$ values and normalized step heights $H_{1} / b_{0}$ is also discussed by demonstrating the velocity distribution in the flow field and the pressure distribution on the surface of a plane boundary. Furthermore, the flow pattern of a synthetic jet near a rigid boundary is compared with that of a continuous jet.

\section{Nomenclature}

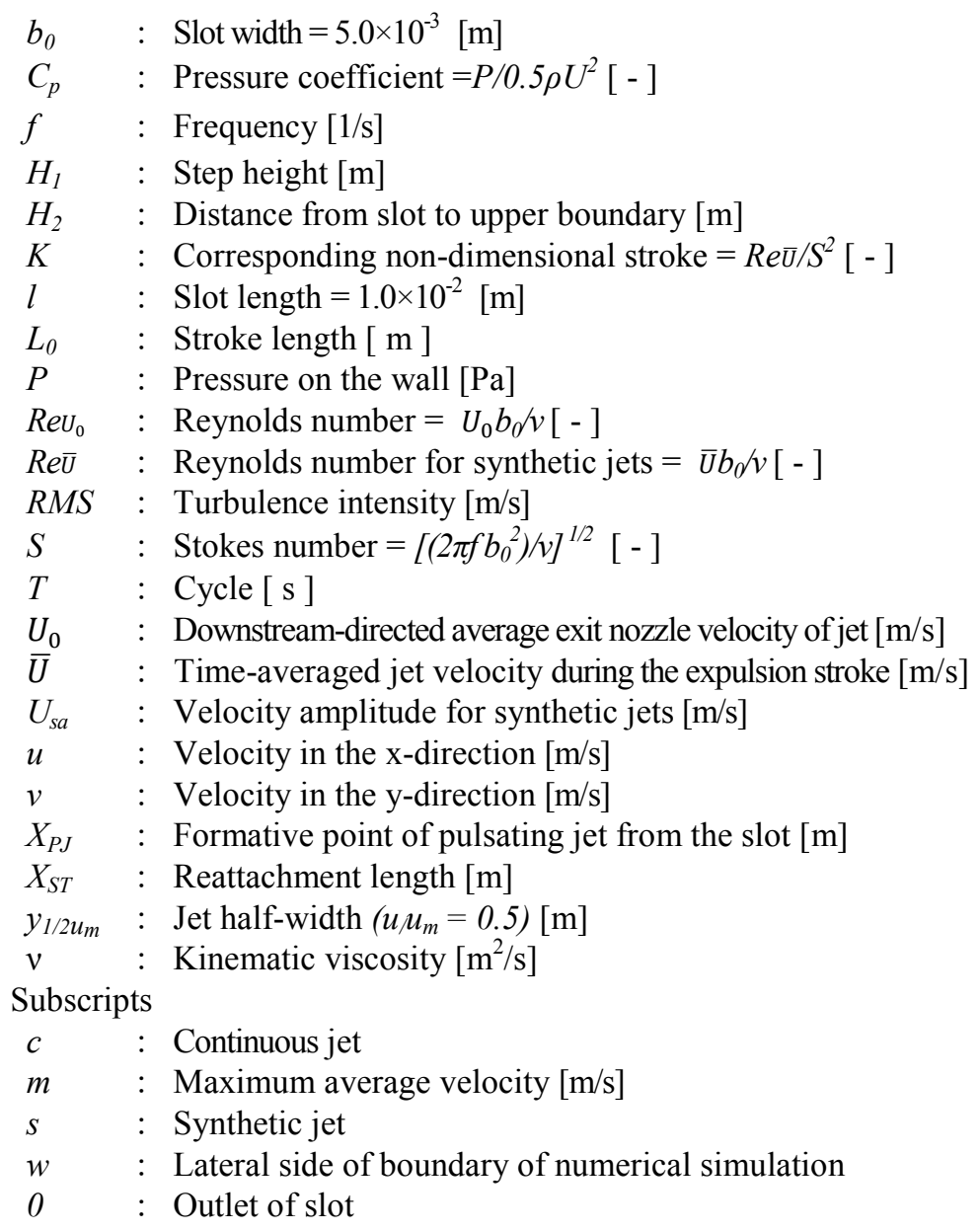

\section{Experimental Setup}

A schematic view of the experimental apparatus is shown in Fig. 1(a). The synthetic jet is generated by a loudspeaker (Diecook D-15L) driven by a signal generator (MCPLG1100D) that sends signals through a power amplifier (Classic Pro V3000). Air is supplied to the continuous jet via the inlet plenum connected to the blower (Showa Denki CO., LTD. U75-2-R313). As illustrated in Fig. 1(a) and 1(b), the rectangular cylinder of the nozzle is supported on each end by an acrylic plate and a two-dimensional jet is induced downstream of the two-dimensional slot. The slot length $l=100 \mathrm{~mm}$, and its width $b_{0}$ can be varied by changing the spacer thickness (in this report, $b_{0}=5 \mathrm{~mm}$, aspect ratio $l / b_{0}=20$ ). The continuous jet and the synthetic jet velocity at the outlet of the slot is controlled by the signal generator and the inverter, respectively. The measurements for the velocities of both jets are performed by a hot-wire anemometer (Kanomax IHW100) with a traverser. (Chuo Precision Industrial ALS-230-C2P). However, the measurements of the flow velocities with 


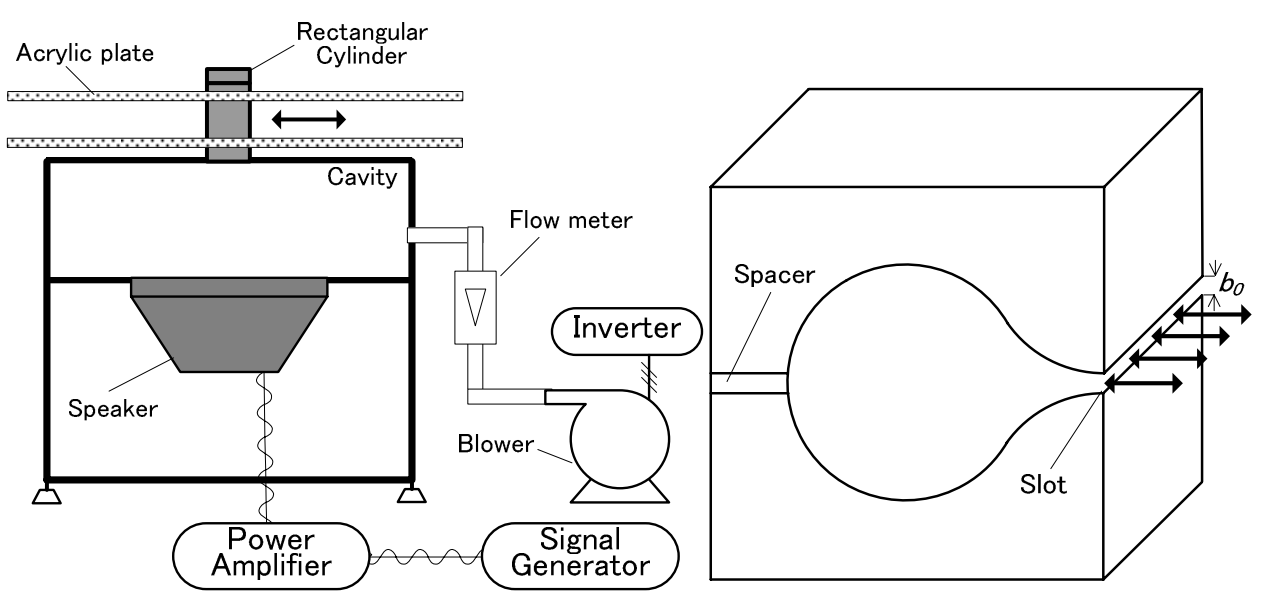

(a) Speaker-driven synthetic jet actuator

(b) Nozzle configuration

Fig. 1 Schematic of experimental apparatus

a hot-wire anemometer are vulnerable to large errors in the regions prone to local backflow, such as the complicated flow field near the slot. A $1 \mathrm{~mm}$ dia. hole was drilled in the rigid wall surface for pressure measurements and a differential pressure manometer (Okano Works, LTD. DMP301N) was used to measure the wall surface pressure distribution.

\section{Numerical Simulation}

A commercially available computer code (SCRYU/ Tetra, Software Cradle Co., Ltd) is used for the numerical simulations. The simulations are conducted by applying the $k-\varepsilon$ turbulent model with approximately 160,000 grid points. The flow conditions correspond to two-dimensional incompressible viscous flow.

Figure 2 depicts a typical numerical domain $\left(l_{x}=400 b_{0}, H_{1}=5 b_{0}, H_{2}=380 b_{0}\right)$ as well as typical boundary conditions. For simplification, the velocity at the inlet, $u_{0}=U_{s a} \sin \omega t$, is applied to the slot instead of the moving boundary condition. In this study, uniform velocity $v_{w}=0.01 U_{S 0}$ and $v_{w}=-0.01 U_{S 0}$ were applied at $H_{1}=380 b_{0}$ and $H_{2}=380 b_{0}$, respectively, which corresponds to the infinite boundary. In case of the continuous jet, $v_{w}=0.01 U_{C 0}$ at $H_{1}=380 b_{0}$ and $v_{w}=-0.01 U_{C 0}$ at $H_{2}=380 b_{0}$ were set as the infinite boundary condition. And constant pressure for the outlet was specified with no-slip conditions at the wall surface for the continuous jet and the synthetic jet.

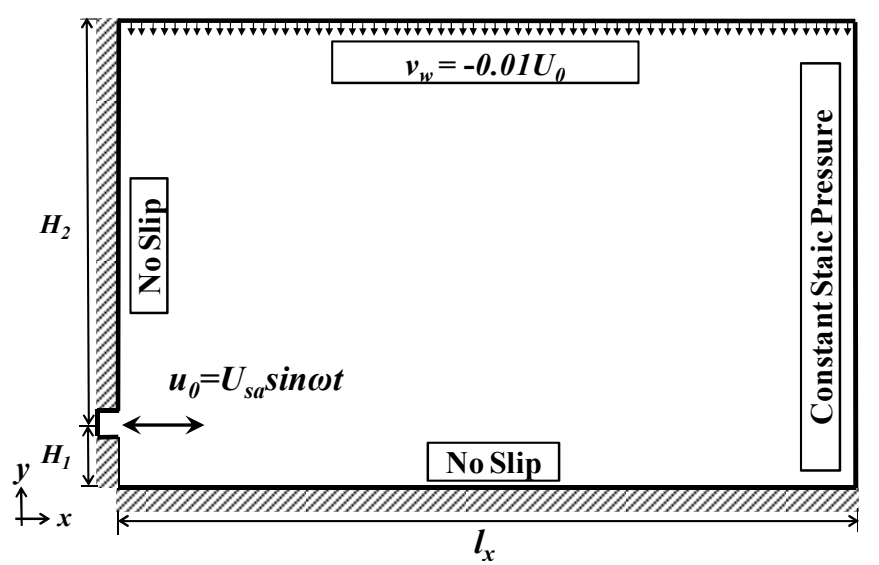

Fig. 2 Numerical simulation domain and boundary conditions

\section{Results and Discussion}

In this paper, two Reynolds numbers are defined in accordance with Holman et al. [2] The first Reynolds number is defined as $\operatorname{Re}_{0}=U_{0} b_{0} / v$ for the continuous jet and the synthetic jet, where $U_{0}$ equals to $U_{C 0}$ is time-averaged velocity for the continuous jet and $U_{0}$ 
equals to $U_{S 0}$ is downstream-directed average exit nozzle velocity of synthetic jet :

$$
U_{s 0}=f L_{0}=\frac{1}{T} \int_{0}^{T / 2} u_{0}(t) d t
$$

The other Reynolds number for synthetic jets is defined as $\operatorname{Re} \bar{U}=\bar{U} b_{0} / v$ to compare to the value used in preceding report [2], where $\bar{U}_{s 0}$ is time-averaged jet velocity during the expulsion stroke:

$$
\bar{U}_{s 0}=\frac{2}{T} \int_{0}^{T / 2} u(t) d t=2 U_{s 0}
$$

A comparison of the flow characteristics between the continuous jet and the synthetic jet is conducted for the same $\operatorname{ReU}_{0}=2430$ condition and a comparison of the flow characteristics between the synthetic jets is conducted for the same $\operatorname{Re} \bar{U}=4860$. Therefore, the jets do not show identical values for momentum. In this experimental apparatus, the frequency of oscillation $f$ is adjustable from $10 \mathrm{~Hz}$ to $50 \mathrm{~Hz}$ under same $\operatorname{Re}_{0}=2430$ and $\operatorname{Re} \bar{U}=4860$. Under these conditions, $f=10 \mathrm{~Hz}$ corresponds to $K=47.76$, and $f=50 \mathrm{~Hz}$ corresponds to $K=9.55$. Therefore, in this study, the results at $K=9.55$ and at $K=47.76$ are used as typical examples of low and high values for $K$, respectively.

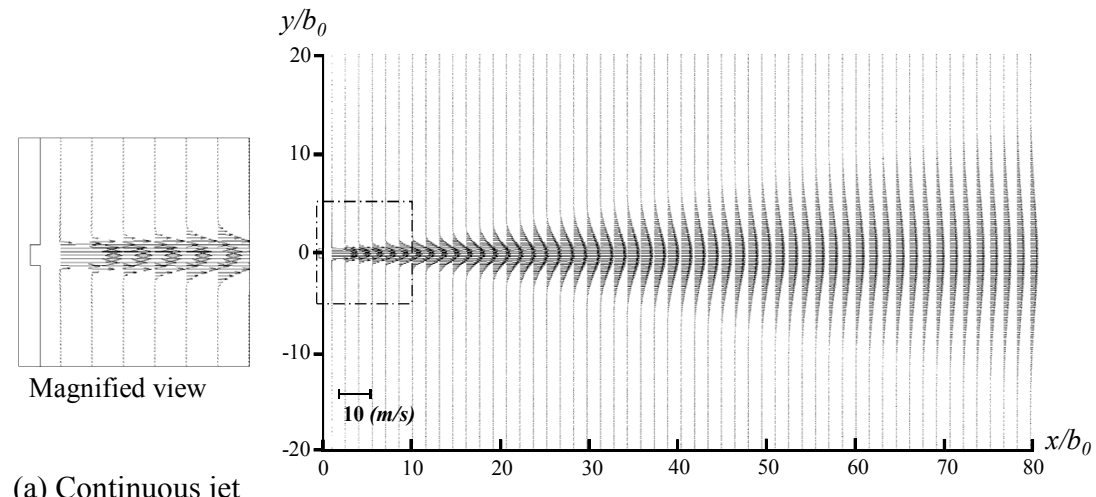

Continuous jet

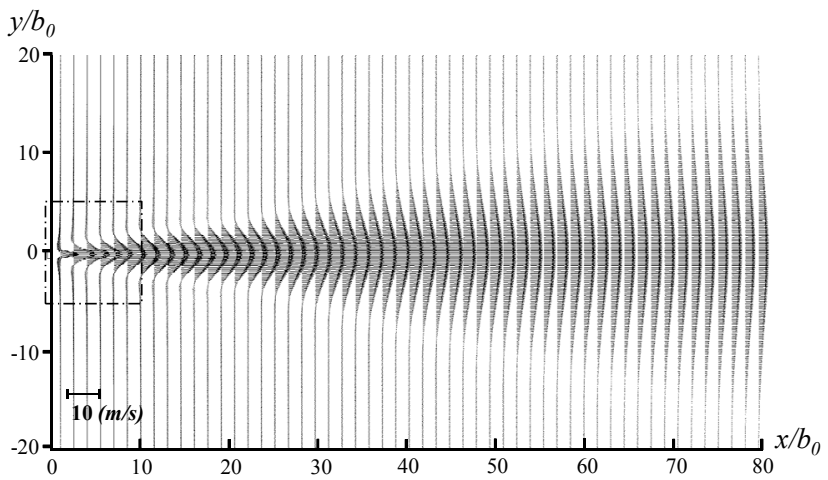

(b) Synthetic jet $(K=9.55, f=50 \mathrm{~Hz})$

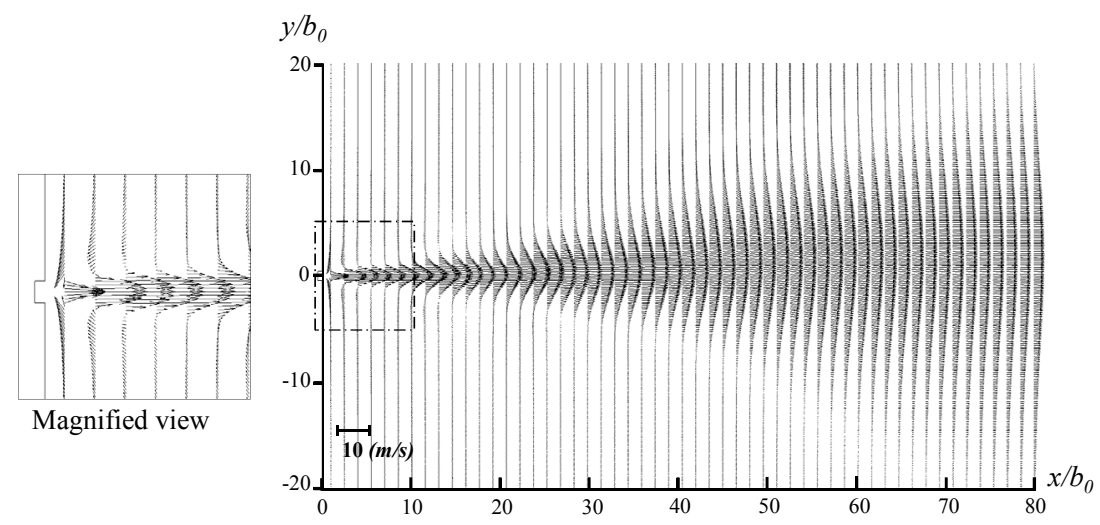

(c) Synthetic jet $(K=47.76, f=10 \mathrm{~Hz})$

Fig. 3 Time-averaged velocity field 


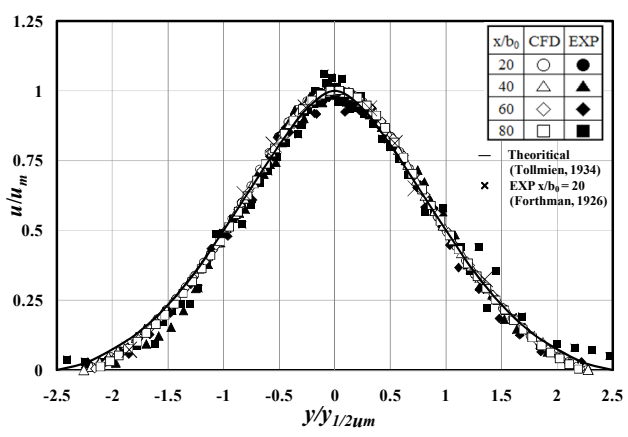

(a) Continuous jet

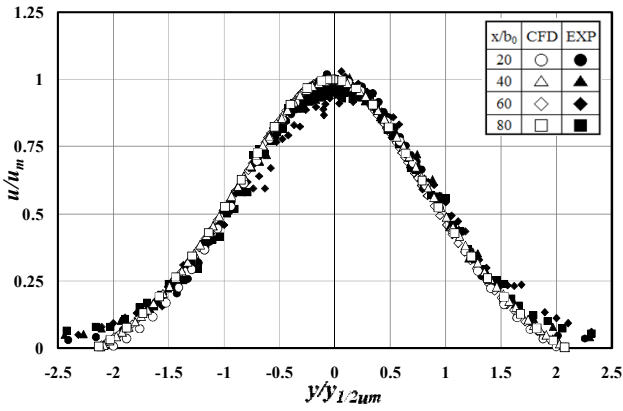

(b) Synthetic jet $(K=9.55, f=50 \mathrm{~Hz})$

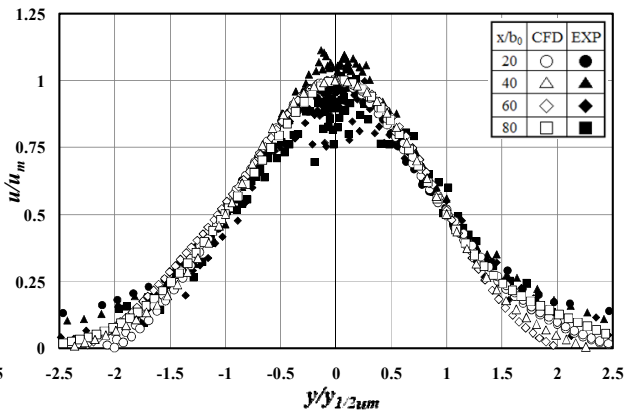

(c) Synthetic jet $(K=47.76, f=10 \mathrm{~Hz})$

Fig. 4 Distribution for two-dimensional velocities of free continuous jet and synthetic jet (EXP: Experiment, CFD: Numerical simulation)

\subsection{Validity of the numerical simulations}

Figure 3 shows typical numerical results of the time-averaged velocity field in the case of $H_{1} / b_{0}=\infty$. Figure 3(a), (b) and (c) show a continuous jet, a synthetic jet with $K=9.55$ $(f=50 \mathrm{~Hz})$ and a synthetic jet with $K=47.76(f=10 \mathrm{~Hz})$, respectively. The figure also shows an expanded view of the nozzle vicinity. The only difference in Fig. 3(b) and (c) is the frequency. At $x / b_{0}>20$, (a), (b) and (c) indicate the same velocity distribution. No backward flow is observed in Fig. 3(a), whereas a clear back flow can be seen near the outlet of the slot in Fig. 3(b) and (c). It is observed that the maximum velocity of the synthetic jet is higher than that of the continuous jet. The jet width of the synthetic jet in Fig. 3(b) and (c) expands in comparison with the width of the continuous jet in Fig. 3(a). Also, the jet width of the synthetic jet in Fig. 3(b) expands more than that in Fig. 3(c) at $x / b_{0}$ $<30$. However, at $x / b_{0}>30$, the jet width in Fig. 3(c) expands exponentially in comparison with that in (b). These results show good agreement with the experimental results of Koso et al. [7]. Therefore, it is concluded that the fundamental flow characteristics of the two-dimensional synthetic jet are highly dependent on $K$.

Figure 4 shows the distribution for the time-averaged velocities of the numerical results compared to those of the experiment in the case of $H_{I} / b_{0}=\infty$, with parameters $x / b_{0}=20,40$, 60 and 80. Figure 4(a), (b) and (c) show the continuous jet, the synthetic jet $(K=9.55)$ and the synthetic jet $(K=47.76)$, respectively. In these figures, the horizontal axis is the ratio of the $y$ distance to the jet half-width $y_{1 / 2 u_{m}}$ and the vertical axis is $u / u_{m}$, where, $u_{m}$ is the maximum average velocity in the center of the jets. For reference, Fig. 4(a) also presents the experimental results from Forthman [8] and the theoretical analysis results from Tollmien [8]. In Fig. 4, the data of the continuous jet (a) and the synthetic jet (b) are distributed generally on one curve, so not much difference exists in the jet velocity distribution for $H_{l} / b_{0}=\infty$. In addition, the experimental and numerical results are in good agreement. These results mean the numerical simulation is valid under the conditions of the present study. In contrast, in the experimental results for $x / b_{0}=60,80$ in the synthetic jet (c), the 
experimental results are not distributed on the curve. The reason is the two-dimensional jet structure collapses downstream for $x / b_{0}>60$; that is, for $x / b_{0}=60,80$ in the synthetic jet (c), it is difficult to compare the results of numerical simulations that are conducted assuming a two- dimensional flow.

\subsection{Comparison of flow characteristics between the continuous jet and the synthetic jet}

Figure 5 shows the growth of the jet half-width $y_{1 / 2 u_{m}}$ of the continuous and synthetic jets downstream of the slot. For comparison, the theoretical analysis results from Tollmien and Goertler [8], and the experimental results from Forthman [8] are also shown. The experimental results correspond with the numerical results. In all cases, the jet half-width expands with the distance downstream of the slot, but the half-widths of the synthetic jets are larger than those of the continuous jet, as shown in Fig. 3. However, the experimental results of the synthetic jets at the outlet of the slot $\left(x / b_{0} \leq 20\right)$ are higher than the numerical results. This is because the back flow is also measured by the hot-wire anemometer. Incidentally, for a jet half-width of $x / b_{0} \leq 20$, the experimental and numerical results for the synthetic jet with $K=9.55$ are higher than those with $K=47.76$. In contrast, for $x / b_{0} \geq 40$, the results with $K=47.76$ are higher than those with $K=9.55$. Therefore, the start point for the expansion of the jet half-width depends on the constant $K$.

Figure 6 shows how the dimensionless maximum streamwise velocities along the

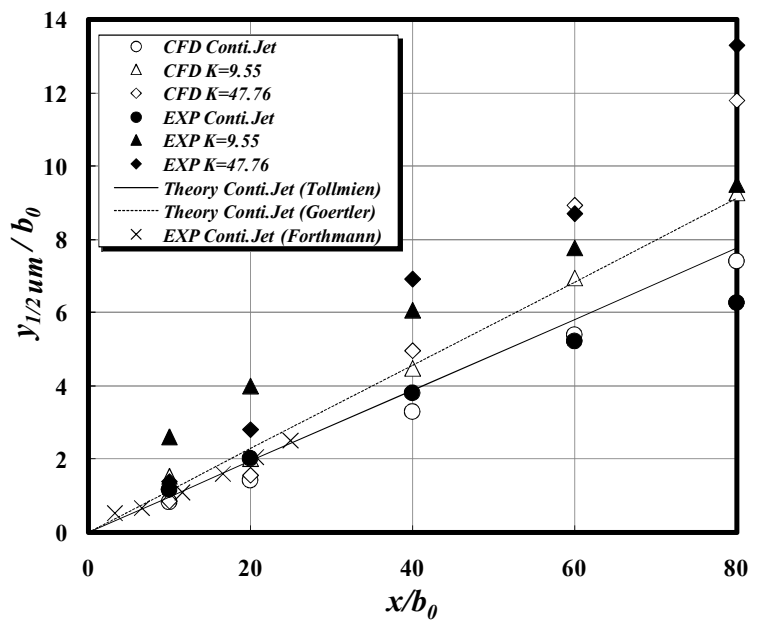

Fig. 5 Growth of the jet half-width for the continuous jet and the synthetic jets (EXP: Experiment, CFD: Numerical simulation)

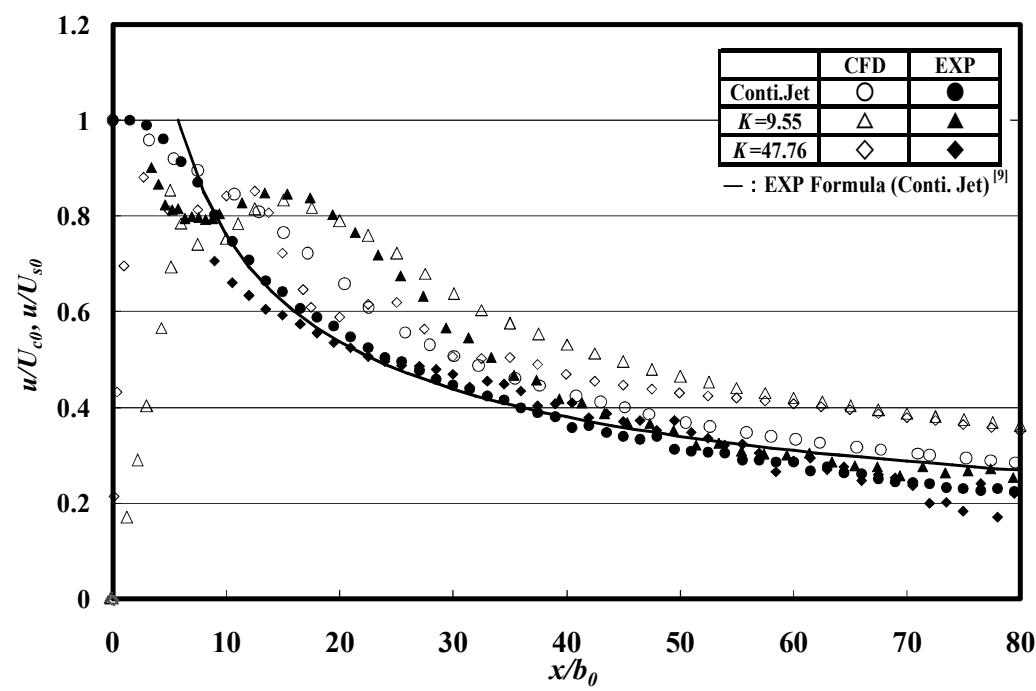

Fig.6 Dimensionless maximum streamwise velocities along the centerline varied with $x / b_{0}$ (EXP: Experiment, CFD: Numerical simulation) 
centerline varied with $x / b_{0}$. Values predicted by the empirically obtained equation [9] are also provided for reference. $u_{m} / U c_{0}$ monotonically decays with respect to the continuous jet as $x / b_{0}$ increases, while $u_{m} / U c_{0}$ shows both increases and decreases with respect to a synthetic jet. The time-averaged velocity of a synthetic jet is 0 at the nozzle outlet, so this means that there must exist some downstream point of maximum velocity, but the stream does not show monotonic decay thereafter. The results of the experiment and of the numerical simulation generally match in a qualitative way in this figure, but there is a quantitative discrepancy; the numerical results tended to exceed the experimental findings, except in the vicinity of the nozzle. This discrepancy was probably due in the most part to the assumption of constant two-dimensional flow in the simulation, while in the experiment, the two-dimensional structure of the jet flow collapsed with increasing distance downstream. Turning to a comparison of the results with $K=9.55$ and $K=47.76$, aside from the nozzle vicinity, i.e., at $x / b_{0}>10$, the predictions with $K=9.55$ showed higher values than those of $K=47.76$. The reader can see that under these experimental conditions, lower values for $K$ resulted in higher values for the maximum velocity along the centerline. However, these flows become complicated, and there remain numerous unclear aspects of the maximum velocities of these flows, including the numerically predicted extreme values. More detailed investigations will be necessary to clarify these issues.

The velocity vector fields of the synthetic jets in the maximum suction cycle in the numerical simulations are shown in Fig. 7. Figure 7(a) shows the synthetic jet for $K=9.55$ and (b) shows the synthetic jet for $K=47.76$. The velocity distribution $(u>0)$ is confirmed in the maximum suction cycle in both (a) and (b), and the suction flow is observed up to

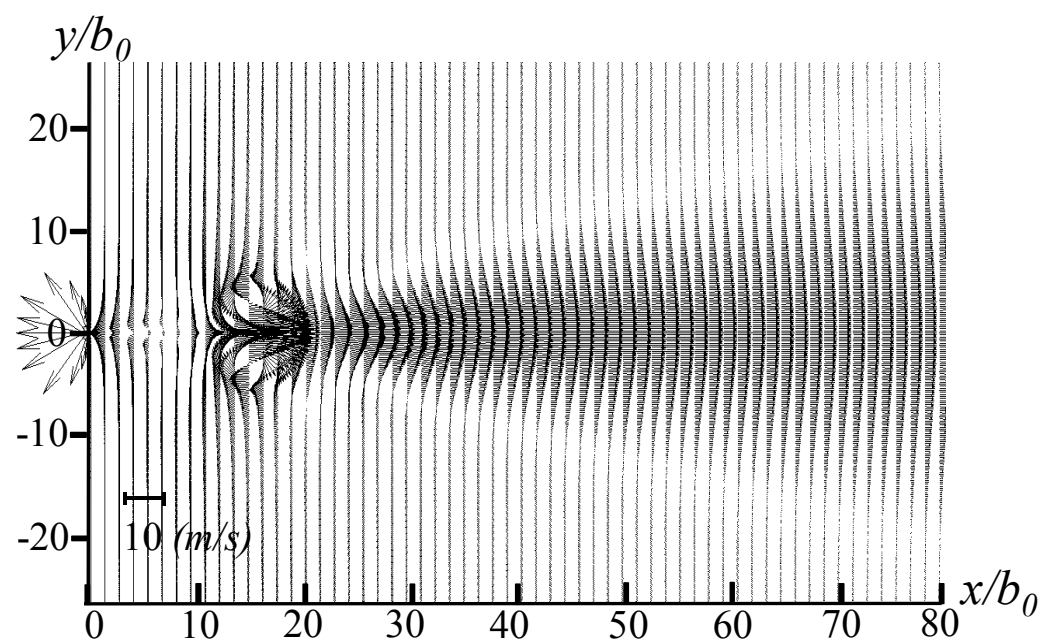

(a) Synthetic jet $(K=9.55, f=50 \mathrm{~Hz})$

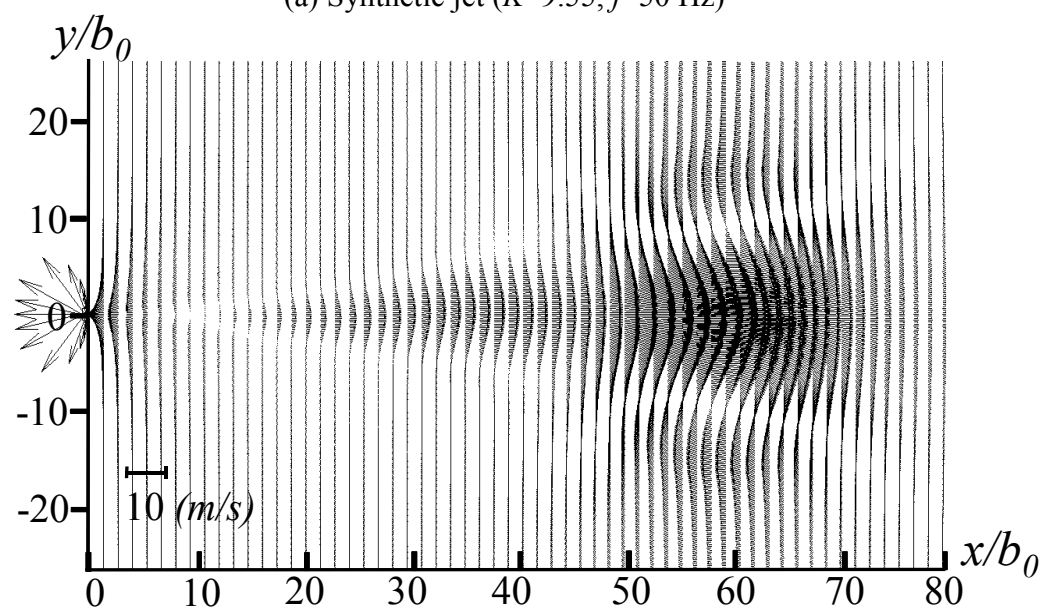

(b) Synthetic jet $(K=47.76, f=10 \mathrm{~Hz})$

Fig. 7 Velocity vector fields in the maximum suction cycle from the simulations 
$x / b_{0} \cong 3$ and $x / b_{0} \cong 8$ on the center of the jets in (a) and (b), respectively. In addition, it can be seen in Fig. 7(a) and (b) that pulse-like jets are produced for $x / b_{0} \geq 3$ and $x / b_{0} \geq 8$, respectively. In Fig. 7(a) a continuous jet develops from $x / b_{0} \cong 25$. However, in Fig. 7(b), despite the downstream $x / b_{0} \geq 50$, the vortex pair does not disappear. Furthermore, the flow in case (b) develops belatedly in comparison with that in case (a). These results suggest that the development of synthetic jets depends on the constant $K$.

Figure 8 shows the formative point of the pulsating jet at the centerline for various values of $K$. The horizontal axis is $K$ and the vertical axis is the dimensionless formative point of the pulsating jet from the slot $\left(X_{P J} / b_{0}\right)$, which is defined as $u=0$ at the maximum suction cycle. The experimental results correspond qualitatively to the numerical results. It is found that $X_{P J} / b_{0}$ from both the experiment and the numerical simulations increases approximately in proportion to the constant $K$.

Figure 9 shows the variation of the turbulence intensity (RMS) along the centerline of the slot. The filled symbols indicate the experimental values, and the open symbols indicate the numerical values. The $R M S$ value in these numerical calculations applied to the $k-\varepsilon$ turbulent model is the sum of the velocity fluctuation component with $\operatorname{Root}(k)$ and is assumed to be the actual value for the velocity fluctuation component. For reference, the experimental results of the continuous jet by Heskestadt [10] are presented. The experimental and numerical results show good qualitative agreement. In the experimental results, the curve of $R M S$ for the continuous jet has a positive slope everywhere. In contrast, the curve for the synthetic jet with $K=9.55$ changes to a positive slope at $x / b_{0}=25$. In this

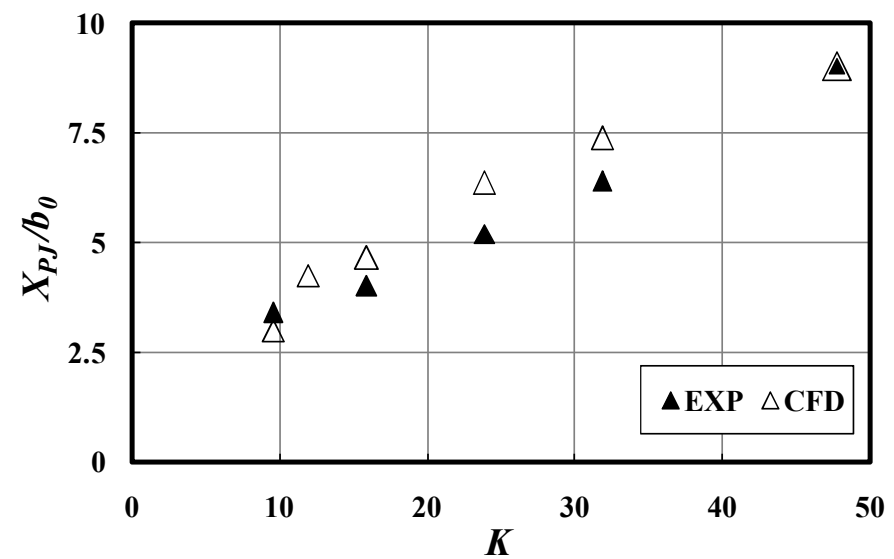

Fig. 8 Formative point of the pulsating jet at the centerline with various values of $K$ (EXP: Experiment, CFD: Numerical simulation)

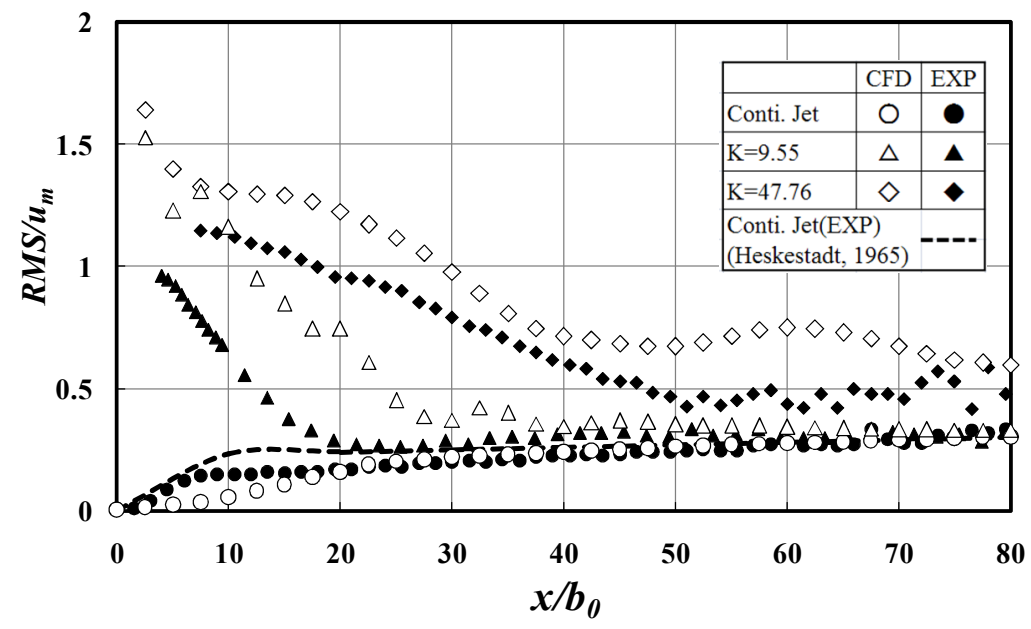

Fig. 9 Variation of RMS values streamwise along the centerline (EXP: Experiment, CFD: Numerical simulation) 
case, the unsteady flow characteristics of the synthetic jet correspond to those of the continuous jet downstream for $x / b_{0}=25$. For $K=47.76, R M S / u_{m}$ generally has a positive slope when $x / b_{0}>52$. The location where $R M S / u_{m}$ has a positive slope, as in the continuous jet, depends on $K$. In the numerical simulation, it is not possible to verify that $R M S / u_{m}$ has a negative slope at $x / b_{0}>30$ for $K=9.55$ or at $x / b_{0}>50$ for $K=47.76$. The positive slope could not be identified in the numerical results under these conditions, as in the experimental results. Although there are qualitative discrepancies between the calculated and the experimental results in these conditions, qualitatively speaking, the flow shows unsteady characteristics resembling those of the continuous flow at an earlier stage, when $K$ is low rather than when $K$ is large. However, in the results from the numerical simulation with $K=$ 47.76 , the trajectory is not the same as the other trajectories. Further research is necessary to clarify the reason for these results.

\subsection{Influence of the rigid boundary on synthetic jets}

A typical time-averaged velocity field near the rigid boundary is shown in Fig. 10 for $H_{1} / b_{0}=5$ and $H_{1} / b_{0}=20$. Figure 10 (i), (ii) and (iii) show the continuous jet, synthetic jet ( $K$ $=9.55)$ and synthetic jet $(K=47.76)$, respectively. Under any conditions, the Coanda effect can be observed. The Coanda effect is found clearly in the continuous jet in both of (a) and (b) and the synthetic jet (ii)(b). In contrast, in case of (ii)(a), (iii)(a) and (iii)(b), it is not clearly seen that the jet flow is bent to the wall. In addition, the half-width of the synthetic jets expands with increasing $x / b_{0}$, as for $H_{l} / b_{0}=\infty$. Furthermore, in (iii)(b), the average curvature for the center of the flow from the slot to the stagnation point on the wall is larger than that in (ii)(b), based on the fact that $X_{P J}$ is further away in case (iii)(b). Therefore, it is

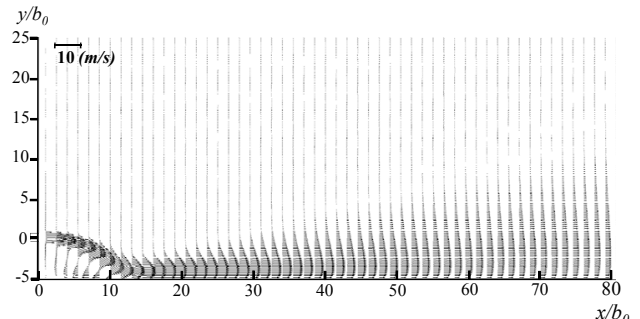

(a) $H_{1} / b_{0}=5$

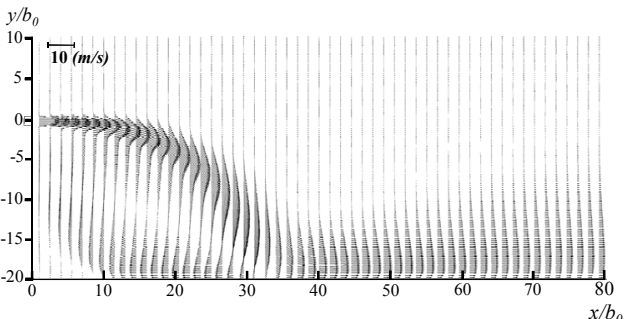

(b) $H_{1} / b_{0}=20$

(i) Continuous jet

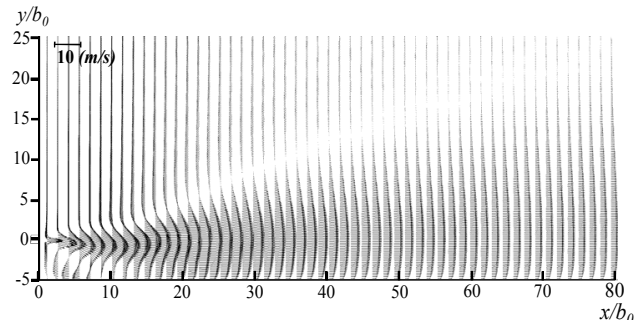

(a) $H_{1} / b_{0}=5$

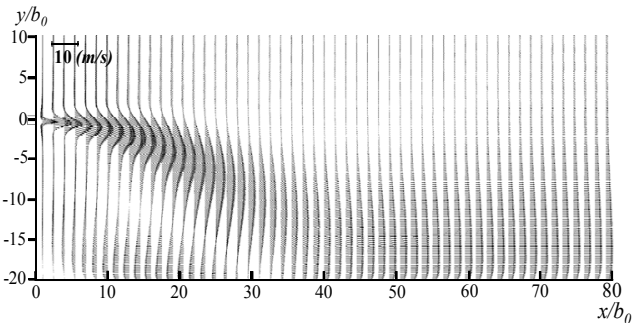

(b) $H_{1} / b_{0}=20$

(ii) Synthetic jet $(K=9.55)$

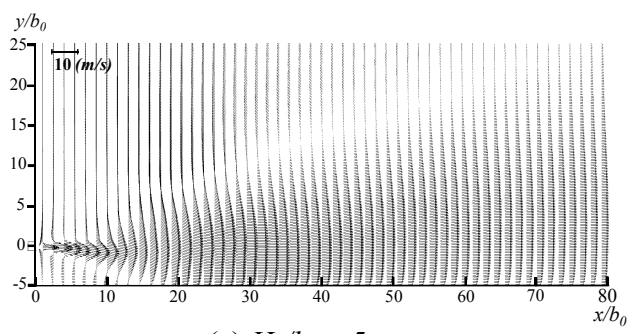

(a) $H_{1} / b_{0}=5$

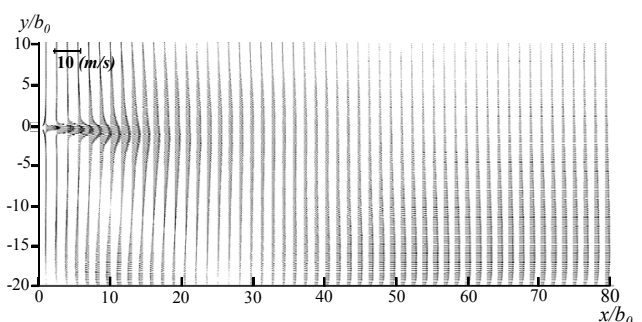

(b) $H_{1} / b_{0}=20$

(iii) Synthetic jet $(K=47.76)$

Fig. 10 Time-averaged velocity field near a rigid boundary 
concluded that the relations between the formative point of the pulsating jet from slot $\left(X_{P J} / b_{0}\right)$, the dimensionless step height $\left(H_{l} / b_{0}\right)$, and the characteristics of the jet half width expansion, determine the re-attachment length $X_{s t}$. In case of the synthetic jet near the rigid boundary, the greater the constant $K$, the more wildly the time variation of the jet velocity fluctuates in the region(s) corresponding to the recirculation zone of the attached continuous jet, and the bending of the jet toward the wall cannot be very clearly visualized in the time-averaged velocity field.

Figure 11 shows the pressure distribution on the rigid wall. The experimental and numerical results for $H_{1} / b_{0}=5$ and $H_{1} / b_{0}=20$ show good agreement qualitatively. The

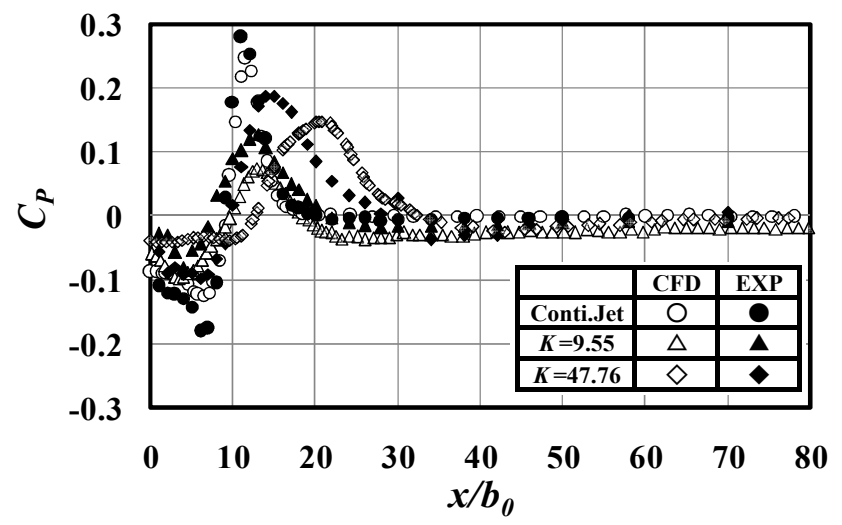

(a) $H_{l} / b_{0}=5$

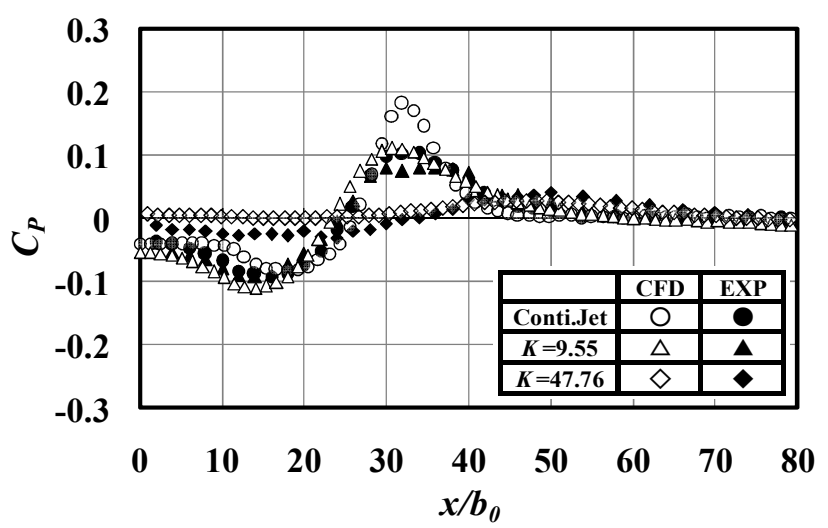

(b) $H_{1} / b_{0}=20$

Fig. 11 Pressure distribution on a rigid wall (EXP: Experiment, CFD: Numerical simulation)

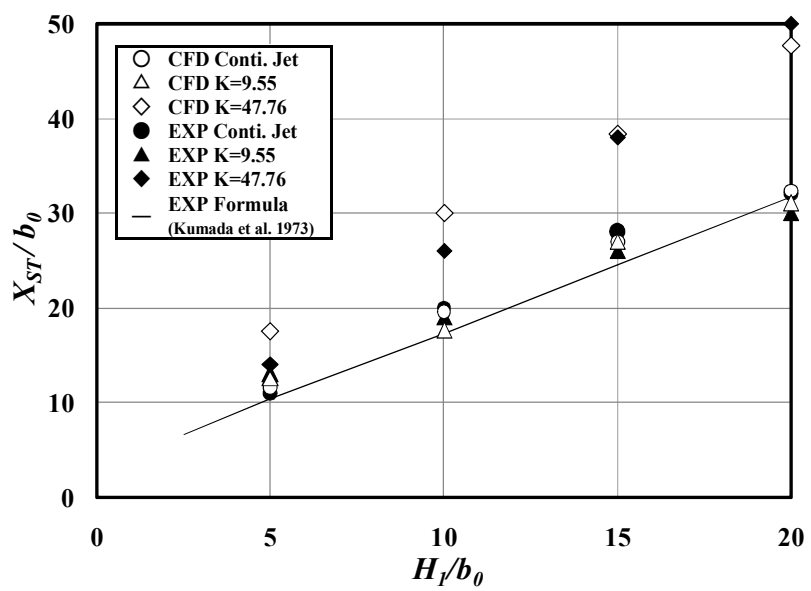

Fig. 12 Stagnation points on the rigid wall (EXP: Experiment, CFD: Numerical simulation) 
strength of the pressure at a stagnation point on the wall is higher for the continuous jet than for the synthetic jets. For the synthetic jets, the strength for $K=9.55$ is higher than that for $K=47.76$. The distance of the stagnation points from the slot $\left(X_{s t} / b_{0}\right)$ denotes the same tendency as that shown by the formative point of the pulsating jet $\left(X_{P_{J} /} b_{0}\right)$ in Figure 8 .

Figure 12 shows the variation of the stagnation points on the rigid wall for various $K$ values. The results of the continuous jet are the same as those by Kumada et al. [11]. The experimental results of the synthetic jet correspond with the numerical results. It is found that $X_{s} / b_{0}$ increases in proportion to $H_{l} / b_{0}$ and this tendency depends on the constant $K$.

\section{Conclusions}

An attempt was made to clarify the fundamental flow characteristics of free synthetic jets $\left(H_{1} / b_{0}=\infty\right)$ and synthetic jets near a rigid boundary by an experiment and numerical simulations. A comparison of the flow characteristics between the continuous and the synthetic jets and the influence of the rigid plane boundary on the behavior of the synthetic jets with various $K$ and $H_{1} / b_{0}$ values were discussed. The following conclusions were obtained.

(1) The experimental results and the numerical simulation results show good qualitative agreement in the comparison of velocity distributions, unsteady flow characteristics, $R M S$, and pressure distributions on the wall. Therefore, the validity of the numerical simulations is confirmed.

(2) Although the expansion of the jet half-widths of the synthetic jets is not the same as that of the continuous jet, the velocity distribution of the synthetic jets indicate similarity of the maximum velocity at the centerline and the jet half-width.

(3) The non-dimensional formative point of the pulsating jet $\left(X_{P J} b_{0}\right)$ from the slot increases approximately in proportion to the constant $K$.

(4) The distribution of $R M S / u_{m}$ along the jet centerline shows unsteady characteristics that resemble those of the continuous flow at an earlier stage, when $K$ is low rather than when $K$ is large.

(5) The flow characteristics of the synthetic jet near the rigid boundary and the re-attachment distance of the synthetic jet depends not only on dimensionless step height $H_{1} / b_{0}$ but also on the constant $K$.

(6) It is shown that the relations between the formative point of the pulsating jet from the slot $\left(X_{P J} / b_{0}\right)$ and the normalized step heights $\left(H_{1} / b_{0}\right)$, and the difference of the jet half-width, determine the length of the re-attachment $\left(X_{s t} b_{0}\right)$.

\section{Acknowledgements}

The authors would like to thank Professor Toshihiko Shakouchi of Mie University and Dr. Shimpei Okayasu of Mitsubishi Heavy Industries, Ltd. for their helpful advice and to Mr. Shunichi Fujiki, the president of Beltek International Corporation for his assistance in the design and construction of the experimental apparatus. This work was carried out with the support of a Grant-in-Aid for Scientific Research (21560187) and a Research Center for Urban Disaster Mitigation (UDM) of Kogakuin University. 


\section{References}

(1) Tensi, J. et al., Modification of the Wake behind a Circular Cylinder by Using Synthetic Jets, Journal of Visualization, Vol.5, No.1, pp.37-44 (2002).

(2) Holman, R et al., Formation Criterion for Synthetic Jets, AIAA Journal, Vol. 43, No.10, pp. $2110-2116$ (2005).

(3) Whitehead, J. and Gursul, I., Interaction of Synthetic Jet Propulsion with Airfoil Aerodynamics at Low Reynolds Numbers, AIAA Journal, Vol. 44, No.8, pp. 1753-1766 (2006).

(4) Zhang, P.F. and Wang, J.J., Novel Signal Wave Pattern for Efficient Synthetic Jet Generation, AIAA Journal, Vol. 45, No.5, pp.1058-1065 (2007).

(5) Shuster, J.M. and Smith, D.R., An Experimental Study of the Formation and Scaling of a Round Synthetic Jet, Physics of Fluids, Vol. 19-045109, pp.1-21 (2007).

(6) Amitay, M. et al., Aerodynamic Flow Control over an Unconventional Airfoil Using Synthetic Jet Actuators AIAA Journal, Vol. 39, No.3, pp.361-370 (2001).

(7) Koso, T. and Kinoshita, T., Jet Flow Formation Using an Annular Synthetic Jet Actuator, JSME annual meeting, pp.211-212 (2006).

(8) Shakouchi, T., Jet Flow Engineering, Morikita Publishing Co., Ltd. (2004).

(9) Rajaratnam, N., Turbulent Jets, Elsevier Scientific Publishing Company, Equation (1.93), (1976).

(10) Heskestadt, G., Hot-Wire Measurements in a Plane Turbulent Jet, Trans. ASME, J.Appl. Mech., Vol. 32, pp.721-734 (1965).

(11) Kumada, M., Mabuchi, I., and Oyakawa, K., Studies on Heat Transfer to Turbulent Jets with Adjacent Boundaries, Transactions of the Japan Society of Mechanical Engineers, 39(319), pp.920-929 (1971). 